Supplement of Solid Earth, 5, 1055-1069, 2014

http://www.solid-earth.net/5/1055/2014/

doi:10.5194/se-5-1055-2014-supplement

(C) Author(s) 2014. CC Attribution 3.0 License.

(c) (i)

Supplement of

\title{
Fully probabilistic seismic source inversion - Part 1: Efficient parameterisation
}

\author{
S. C. Stähler and K. Sigloch
}

Correspondence to: S. C. Stähler (staehler@geophysik.uni-muenchen.de) 


\title{
Supplement to "Fully probabilistic seismic source inversion - Part 1: Efficient parametrisation"
}

\author{
S. C. Stähler and K. Sigloch
}

\begin{abstract}
Martin Vallée raised an important point in the discussion stage of this paper. The choice of the orthogonal functions is obviously biased by our database. In our database, we find almost no event with a "slow start characteristic". This might be due to the selection process employed in Sigloch \& Nolet (2006). However, we do believe that the absence of "slow start events" is real. In the P-waveforms, we often see a small first arriving peak, followed by a strong secondary arrival. However, in modeling the waveforms this can usually be explained by the radiation pattern. The small first peak is $\mathrm{P}$, the stronger second one pP. So we see almost no "slow start events" in our database.

It still might be the case that we excluded them all for some reason, so it might be interesting to see how our orthogonal functions fare in expressing these late peaks. For that we constructed several test functions we considered difficult to fit.
\end{abstract}

1. Fig.1 shows a 5 s rise time triangular STF. Fitting with 8 base functions adds some ringing to the signal. Above 12 base functions the fit is reasonable.

2. Fig. 2 shows a 5 s rise time triangular STF, happening after 10 s of silence. This shape is rather poorly fit by 12 of our base functions, notice the strong ringing in the beginning of the time series. However, this solution (absolutely 0 before a rather benign STF) is discouraged by the algorithm, since it allows for individual shifting of the waveforms.

3. Fig. 3 shows a similar STF, but now with a slow rise to $50 \%$ amplitude before the triangle. This shape can be rather well expressed by 12 to 16 base functions.

4. Fig.4 shows the same, but with a slower rise. The 12 wavelet-representation shows a bit more ringing on the slow slope in the beginning.

All in all, we think that the level of fit between the test functions and the 12 waveletrepresentation is acceptable even for those difficult waveforms. However, it might be beneficial to allow for 16 or more base functions, if the fit is poor with 12 . 


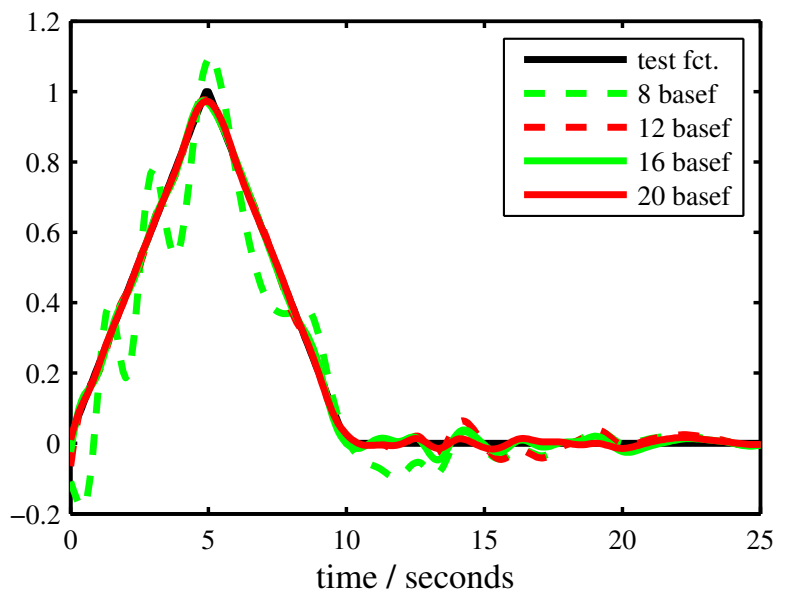

Figure 1: triangle with rise time $5 \mathrm{~s}$

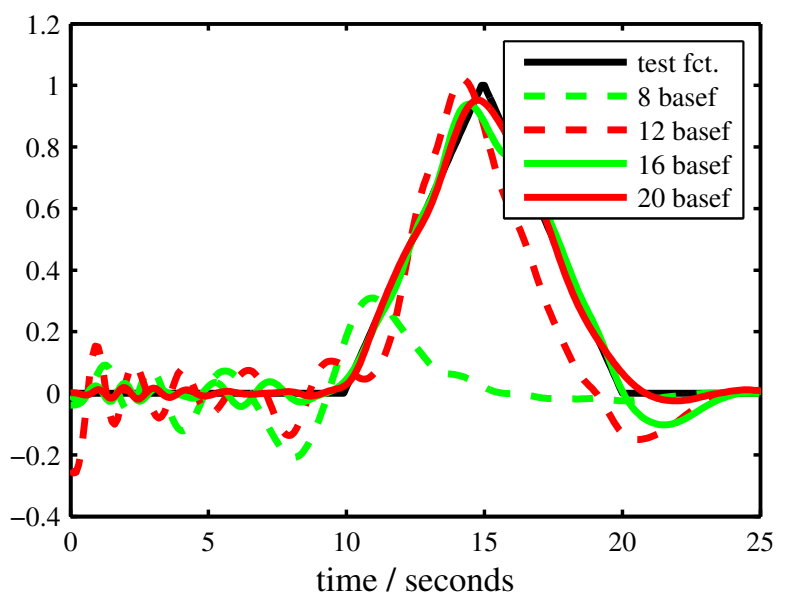

Figure 2: triangle with rise time $5 \mathrm{~s}$, starting after $10 \mathrm{~s}$ 


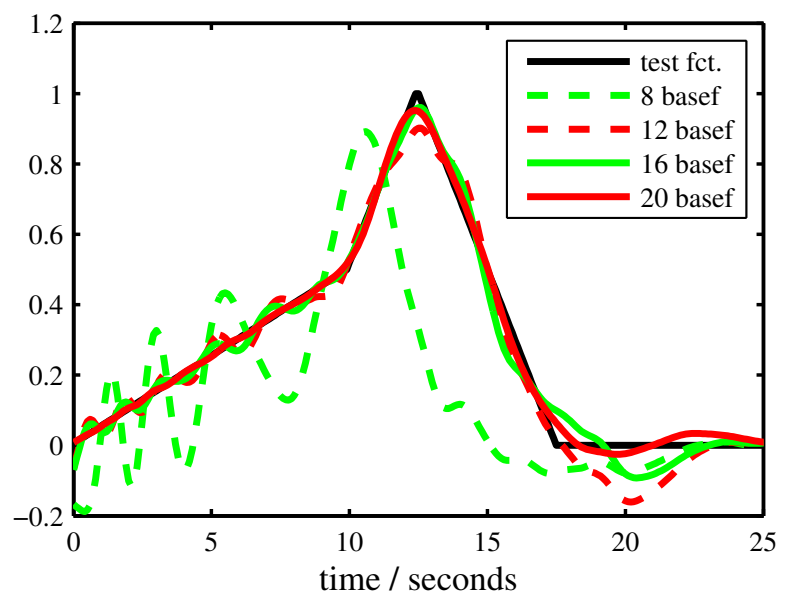

Figure 3: 10 second rise to $50 \%$, then triangle with 5 s rise time

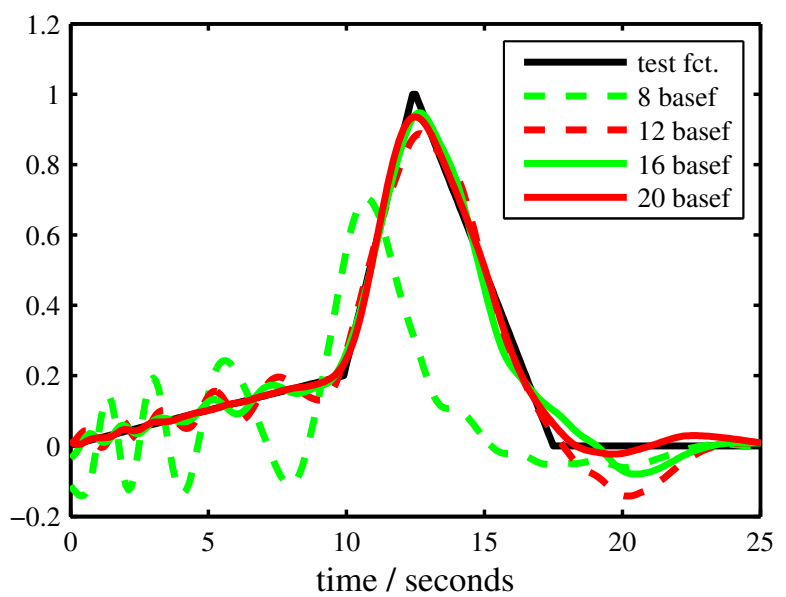

Figure 4: 10 second rise to $20 \%$, then triangle with 5 s rise time 